\title{
ON HOW ENERGY CONSERVATION MIGHT PREVENT BLACK HOLE EXPLOSIONS AND VIOLATIONS OF THE THIRD LAW
}

\author{
R. TORRES ${ }^{1}$ \\ Department of Applied Physics, UPC, Barcelona, Spain. \\ F. FAYOS ${ }^{2}$ \\ Department of Applied Physics, UPC, Barcelona, Spain.
}

\begin{abstract}
We claim that imposing energy conservation to the emission of Hawking radiation and to the modelling of black hole evaporation might prevent black hole explosions as well as violations of the third law of black hole thermodynamics. This is specifically shown for the general class of spherically symmetric quantum black holes described by an effective quantum vacuum invariant under boosts in the radial direction. No assumptions are made with regard to the specific framework from which the quantum black holes are derived.
\end{abstract}

Keywords: Black Holes; Hawking radiation; Black Hole Thermodynamics; Third Law. PACS numbers: 04.70.Dy; 04.70.-s

\section{INTRODUCTION}

The celebrated result that black holes $(\mathrm{BH})$ radiate a thermal spectrum of particles was first derived [1] using quantum field theory on a fixed curved background (Schwarzschild's solution). In this model the black hole temperature was proportional to the inverse of the black hole mass. In this way, if one followed the evolution of the black hole as it emits radiation with its consequent mass loss, one would expect a final event in which the temperature and the luminosity would diverge, indicating a final black hole explosion. Of

\footnotetext{
${ }^{1}$ E-mail: ramon.torres-herrera@upc.edu

${ }^{2}$ E-mail: f.fayos@upc.edu
} 
course, one would go too far in deducing that real black holes explode from such a simple model. For instance, it lacks of the quantum gravity corrections that one expects to appear as the last stages of black hole evaporation are reached. Nowadays, different approaches to quantum gravity exist. Some of the models derived from them suggest a final BH explosion while some others challenge this picture. In the later case, it is usual that the final explosion is avoided by reducing Hawking radiation when the black hole mass is close to a critical mass of the order of Planck's mass. This could represent an extra problem since a black hole that effectively reached this critical value would be in absolute zero temperature. In this way, such a black hole would violate the third law of BH thermodynamics that, in its Nerst version, states that absolute zero temperature cannot be reached by a finite number of steps (i.e., by any physical process).

In this paper we propose to use another approach to black hole explosions and the problem of the violations of the 3rd law. It is based in the fact that the procedure applied in [1] was not in agreement with energy conservation, since the energy radiated by the black hole cannot be balanced by a corresponding decrease of its mass if a fixed background is assumed. On the contrary, it was shown in [2] that Hawking radiation can be derived taking into account the back-reaction effect of the radiation on the black hole thanks to the requirement of energy conservation.

Therefore, our aim in this paper will be to analyze the relevance of energy conservation in the last stages of black hole evaporation by following the procedure proposed in [2]. We will try to show that energy conservation might be sufficient to prevent black hole explosions and violations of the third law. The approach will be rather general since a definitive quantum gravity theory is not known. In this way, the proof will be carried out for general effective spherically symmetric spacetimes describing a quantum vacuum invariant under radial boosts, so that the results will be directly applicable for effective spacetimes coming from many different frameworks (see, for example, [4][5][6][7][8][9]).

The paper has been divided as follows. In section 2 we introduce the effective metric describing the black hole and we describe the $\mathrm{BH}$ properties from a strictly thermal point of view. This will allow us to model the thermal evaporation of the black hole and to analyze its possibilities of either ending in a final explosion or violating the third law of $\mathrm{BH}$ thermodynamics. In section 3 we study the black hole under the approach of energy conservation. This will lead us to a different black hole luminosity that will later be used to model the evaporation process under the requirement of energy conservation. The subsequent $\mathrm{BH}$ evolution will be then contrasted with the thermal evolution found in the previous section. Finally, the results are discussed in section 4. 


\section{BH EXPLOSIONS AND VIOLATIONS OF THE 3RD LAW IN THE THERMAL APPROACH}

Let us consider an isolated spherically symmetric black hole in vacuum that is completely characterized by its mass in an effective asymptotically flat spacetime. The general effective metric for a spherically symmetric quantum vacuum invariant under boosts in the radial direction [3] can be written (once a dimensional reduction, if necessary, has been performed) $\mathrm{as}^{3}$

$$
d s^{2}=-f(R ; M) d t_{S}^{2}+f(R ; M)^{-1} d R^{2}+R^{2} d \Omega^{2},
$$

where the dependence $f(R ; M)$ indicates the fact that for every ADM mass $M$ there is a specific effective metric. One can find this form for the effective metric coming from many different approaches to Quantum Gravity [4][5][6][7][8][9]. Since we want to treat the general case comprising all reasonable effective metrics with the form (2.1), we will be less restrictive than usual and we will just assume that $f(R ; M)$ is, at least, a $C^{1}$ function $^{4}$ in the effective space-time. We will also be less restrictive than usual by allowing the possibility to the effective spacetime to be singular, although only at $R=0$, what will allow us to contemplate a wider range of behaviours.

As is well-known, the existence of horizons requires $f(R ; M)=0$. Let us assume that for a given black hole mass there is an outer horizon $R_{+}$. Then, as result of a standard Euclidean continuation of the geometry through $R_{+}$, one gets that the outer horizon emits Hawking radiation with a thermal distribution of temperature

$$
T=\frac{\kappa}{2 \pi},
$$

where $\kappa$ is the surface gravity corresponding to the outer horizon

$$
\left.\kappa=\frac{1}{2} \frac{d f}{d R}\right\rfloor_{R=R_{+}} .
$$

An interesting possibility for quantum black holes that we will also take into consideration in this paper is that the black hole could have a critical mass:

\footnotetext{
${ }^{3}$ Let us remark that there are proposals for effective metrics representing spherically symmetric black holes that are not invariant under boosts in the radial direction (i.e., cannot be written in the form (2.1)). However, it can be shown that the main results in this paper can also be applied to them. As we will see, this is due to the physics behind our results which is independent of the detailed calculations.

${ }^{4}$ Note that we will need the existence of a well-defined surface gravity (2.3) and, thus, a first derivative of $f$ at $R_{+}$. Let us also remind that the usual assumption would be that $f$ is $C^{2}$ so that the effective energy-momentum tensor obtained through Einstein's equations would be defined at every point [10].
} 
Definition: A black hole has a critical mass $M=M_{c r}(\geq 0)$ if: i) the surface gravity of its outer horizon is zero and ii) the specific effective metric from which it comes has no horizon for $M<M_{c r}$.

Thus, the temperature of the outer horizon for a $\mathrm{BH}$ with critical mass would be zero. Moreover, $f^{\prime}\left(R_{+} ; M<M_{c r}\right)$ (where the prime denotes derivative with respect to $R$ ) would not be defined. Note that many quantum black holes possessing critical masses and coming from many different frameworks have been proposed in the current literature (see, for example, [5][6][7][8][9]. We have added a short appendix (A) with a couple of examples for the reader not familiarized with this possibility).

However, let us recall that if the $\mathrm{BH}$ possesses an outer horizon with its corresponding non-zero temperature, this implies a standard thermal distribution for the emitted photons

$$
<n(E)>_{\text {Stand. }}=\frac{1}{\exp (E / T)-1},
$$

where $E$ is the photon energy, and a total flux of radiated energy [11] approximately given by

$$
L_{\text {Stand. }} \simeq \frac{1}{2 \pi} \int_{0}^{\infty}<n(E)>_{\text {Stand. }} \gamma_{E 0} E d E,
$$

where $\gamma_{E 0}$ is the greybody factor and its subindex ' 0 ' indicates that here we only consider the main contribution to the grey-body factor that comes from the zero angular momentum $l=0$ [12]. It can be shown (see, for example, [12]) that (without taking into account energy conservation) for any static spherically symmetric black hole with outer horizon $R_{+}$, and whenever $E M \ll 1$, the greybody factor takes the form

$$
\gamma_{E 0} \simeq 4 E^{2} R_{+}^{2}
$$

Therefore, the luminosity can be approximately expressed as

$$
L_{\text {Stand. }} \simeq \frac{1}{2 \pi} \int_{0}^{\infty} \frac{4 E^{3} R_{+}^{2}}{\exp (2 \pi E / \kappa)-1} d E=\frac{1}{120 \pi} R_{+}^{2} \kappa^{4} .
$$

\subsection{BACKREACTION: Modeling BH EVAPORATION}

In order to model the backreaction produced by the emission of radiation, let us first write the effective metric (2.1) in terms of ingoing Eddington-Finkelstein-like coordinates $\{u, R, \theta, \varphi\}$, where

as

$$
u=t_{S}+\int^{R} \frac{d R^{\prime}}{f(R ; M)},
$$

$$
d s^{2}=-f(R ; M) d u^{2}+2 d u d R+R^{2} d \Omega^{2} .
$$


Now, we can model the mass lost taking into account the heuristic picture that describes Hawking radiation as due to a tunneling process. I.e., whenever a pair of virtual particles is created, when the particle with positive energy escapes to infinity its companion, with negative energy, falls into the black hole thus reducing the BH mass. In this way, if we consider negative energy massless particles following ingoing null geodesics $u=$ constant, the mass at infinity of the black hole becomes a decreasing function $M(u)$. The metric which incorporates the effect of the decreasing BH mass due to the ingoing null radiation is (2.8) with $f(R ; M)$ replaced by $f(R ; M(u))[=f(R, u)]$

$$
d s^{2}=-f(R ; M(u)) d u^{2}+2 d u d R+R^{2} d \Omega^{2} .
$$

On the other hand, the flux of negative energy particles directed towards the black hole equals the flux of outgoing radiated particles that reach the future lightlike infinity and, therefore,

$$
\frac{d M}{d u}=-L_{\text {Stand. }}(M)
$$

\subsection{BlaCK hole COMPlete EVAPORATiON AND EXPlOSiONS}

If the effective metric is such that the outer horizon exists for all $M>0$ with a non-zero surface gravity, then (2.7) inform us that an evaporating black hole has to emit radiation as long as it has mass. On the other hand, close to zero mass (no BH), the outer horizon $R_{+}$has to approach zero. The behaviour of the BH near zero mass depends on the specific behaviour of $\kappa$ or, alternatively, $f^{\prime}\left(R_{+}(M) ; M\right)$. In this way, we have the following

Proposition 1 Let us consider an effective metric whose outer horizon exists for all $M>0$ with a non-zero surface gravity. If the metric satisfies around its zero mass

$$
\left.f^{\prime}\right\rfloor_{R_{+}} \simeq a M^{\alpha} \text { and } R_{+} \simeq b M^{\beta}
$$

where $\beta>0$ and $2 \beta+4 \alpha<1$, then a thermally evaporating black hole modelled with this metric will reach the total evaporation in a finite time.

First, note that the requirement $\beta>0$ comes from the fact that the outer horizon has to approach zero as the BH completely evaporates. Now, to show the proposition it is enough to use (2.11) in (2.7) in order to evaluate (2.10). Then one directly gets that only if $2 \beta+4 \alpha<1$ zero mass is reached for a finite time. On the other hand, this together with the condition $\beta>0$ implies that a necessary condition for the total evaporation to occur is $\alpha<1 / 4$.

One can further consider the subcase in which this evaporation is explosive, meaning that the luminosity approaches infinity as the mass tends to zero. 
Corollary 1 For a thermally evaporating black hole satisfying (2.11) with $\beta+2 \alpha<0$ the evaporation ends in a final explosion.

What comes directly from (2.7). Note that this together with the condition $\beta>0$ imply that the necessary condition for the explosion to occur is $\alpha<0$. Nevertheless, as far as we know, in the models that one finds in the literature $\alpha$ satisfies

$$
\alpha \geq-1
$$

Therefore, from now on we will assume that this inequality is satisfied for the models in this paper. It is interesting to notice that the minimum value that one finds for $\alpha$ corresponds to the standard evaporation of a Schwarzschild black hole in which $\alpha_{S c h}=-1\left(\beta_{S c h .}=1\right)$.

\subsection{ViOLATIONS OF THE 3RD LAW}

If the effective metric is such that the surface gravity of the outer horizon $\kappa$ is zero for a mass $M=M_{c r}>0$, then (2.7) inform us that an evaporating black hole with initial mass bigger that $M_{c r}$ has to emit radiation while approaching its critical mass. The behaviour of the $\mathrm{BH}$ when its mass is close to $M_{c r}$ depends on the specific behaviour of $\kappa$ or, alternatively, $\left.f^{\prime}\right\rfloor_{R_{+}}$as they approach zero. In this way we have the following

Proposition 2 Let us consider an effective metric possessing an outer horizon whose surface gravity is zero for a mass $M=M_{c r}>0$ while $R_{+}\left(M \geq M_{c r}\right)>0$. If the metric satisfies around the critical mass

$$
\left.f^{\prime}\right\rfloor_{R_{+}} \simeq a\left(M-M_{c r}\right)^{\alpha},
$$

where $0 \leq \alpha<1 / 4$, then a black hole whose thermal evaporation from $M>M_{c r}$ were modeled with this metric would violate the 3rd law of black hole thermodynamics.

First, note that the requirement $\alpha \geq 0$ has to be imposed since we assume that $f$ is $C^{1}$ in $R>0$. Then, using (2.12) in (2.7) in order to evaluate (2.10) it is easy to see that the mass reaches the critical value for a finite $u$ if $\alpha<1 / 4$. In other words, it reaches zero temperature (2.2) in a finite time, what would represent a violation of the third law of black hole thermodynamics.

\section{ENERGY CONSERVATION APPROACH}

Let us now consider Hawking radiation coming out from a quantum black hole thanks to the tunneling process occurring through the outer horizon $R_{+}$and taking into account the 
consequences of energy conservation. In order to do this, we will rewrite the effective metric (2.1) in Painlevé-like coordinates [13] so as to have coordinates which are not singular at the horizon. It suffices to introduce a new coordinate $t$ replacing the Schwarzschild-like time $t_{S}$ such that $t=t_{S}+h(R)$ and fix $\mathrm{h}(\mathrm{R})$ by demanding the constant time slices to be flat. In this way one gets:

$$
d s^{2}=-f(R ; M) d t^{2}+2 \sqrt{1-f(R ; M)} d t d R+d R^{2}+R^{2} d \Omega^{2},
$$

In these coordinates the radial null geodesics describing the evolution of test massless particles are given by

$$
\frac{d R}{d t}= \pm 1-\sqrt{1-f(R ; M)}
$$

with the upper (lower) sign corresponding to outgoing (ingoing, respectively) geodesics.

In [14][15][2][16] it was found that, when a self-gravitating shell of energy $E$ travels in a spacetime characterized by an ADM mass $M$, the geometry outside the shell is also characterized by $M$, but energy conservation implies that the geometry inside the shell is characterized by $M-E$. It was also found that the shell then moves on the geodesics given by the interior line element. In this way, according to (3.2), one expects a shell of energy $E$ to satisfy the evolution equation

$$
\frac{d R}{d t}= \pm 1-\sqrt{1-f(R ; M-E)} .
$$

Let us now consider pair production occurring just beneath the event horizon with a positive energy particle tunneling out. The standard results of the WKB method for the tunneling through a potential barrier that would be classically forbidden can be directly applied due to the infinite redshift near the horizon [2]. In particular, the semiclassical emission rate will be given by $\Gamma \sim \exp \{-2 \operatorname{Im} S\}$, where $S$ is the particle action. Therefore, we have to compute the imaginary part of the action for an outgoing positive energy particle which crosses the horizon $R_{+}$outwards from $R_{\text {in }}$ to $R_{\text {out }}$.

$$
\operatorname{Im} S=\operatorname{Im} \int_{R_{\text {in }}}^{R_{\text {out }}} p_{R} d R=\operatorname{Im} \int_{R_{\text {in }}}^{R_{\text {out }}} \int_{0}^{p_{R}} d p_{R}^{\prime} d R .
$$

Using Hamilton's equation $\left.\dot{R}=+d H / d p_{R}\right\rfloor_{R}$ and $H=M-E^{\prime}$, this can be written with the help of (3.3) as

$$
\begin{aligned}
\operatorname{Im} S & =\operatorname{Im} \int_{M}^{M-E} \int_{R_{\text {in }}}^{R_{\text {out }}} \frac{d R}{\dot{R}} d H= \\
& =\operatorname{Im} \int_{0}^{E} \int_{R_{\text {in }}}^{R_{\text {out }}} \frac{d R}{1-\sqrt{1-f\left(R ; M-E^{\prime}\right)}}\left(-d E^{\prime}\right) .
\end{aligned}
$$


If we define

$$
g\left(R_{+} ; M-E^{\prime}\right)=\left.\frac{\partial f\left(R ; M-E^{\prime}\right)}{\partial R}\right|_{R=R_{+}\left(M-E^{\prime}\right)},
$$

where $R_{+}\left(M-E^{\prime}\right)$ is the position of the outer horizon when the $\mathrm{BH}$ mass at infinity is $M-E^{\prime}$, then by deforming the contour of integration so as to ensure that positive energy solutions decay in time and taking into account that a particle just inside the horizon tunnels just outside a shrunken horizon $\left(R_{\text {in }}>R_{\text {out }}\right)$ one gets

$$
\int_{R_{\text {in }}}^{R_{\text {out }}} \frac{d R}{1-\sqrt{1-f\left(R ; M-E^{\prime}\right)}}=-i \pi \frac{2}{g\left(R_{+} ; M-E^{\prime}\right)} .
$$

We can then write $(3.5)$ as

$$
\operatorname{Im} S=\int_{0}^{E} \frac{2 \pi}{g\left(R_{+} ; M-E^{\prime}\right)} d E^{\prime} .
$$

Tunneling also happens when a pair is created outside the horizon and the negative energy particle tunnels into the black hole. Then, following the procedure for the Schwarzschild case in [2], the imaginary part of the action for this ingoing particle satisfies

$$
\operatorname{Im} \int_{0}^{-E} \int_{R_{\text {out }}}^{R_{\text {in }}} \frac{d R}{-1+\sqrt{1-f\left(R ; M-E^{\prime}\right)}} d E^{\prime}=\int_{0}^{E} \frac{2 \pi}{g\left(R_{+} ; M-E^{\prime}\right)} d E^{\prime}
$$

what coincides with the result for the previous channel (3.6). Both channels contribute to the rate of the Hawking process, but we have seen that both contributions provide us with the same exponential term for the semiclassical rate

$$
\Gamma \sim e^{-2 \operatorname{Im} S}=\exp \left(-4 \pi \int_{0}^{E} \frac{d E^{\prime}}{g\left(R_{+} ; M-E^{\prime}\right)}\right) .
$$

When quadratic terms are neglected we can develop $\operatorname{Im} S$ up to first order in $E$ as

$$
\operatorname{Im} S \simeq-\frac{2 \pi}{g\left(R_{+} ; M\right)} E
$$

obtaining a thermal radiation for the black hole $(\Gamma \sim \exp \{-E / T\})$ with temperature

$$
\left.T=\frac{g\left(R_{+} ; M\right)}{4 \pi}=\frac{1}{4 \pi} \frac{\partial f}{\partial R}\right\rfloor_{R=R_{+}}
$$

that coincides with the expected temperature (2.2). 


\subsection{SPECTRAL DistribUtion AND LUMINOSITY}

Notwithstanding the comments about the temperature of the black hole, it is important to remark that the higher order terms in $E$, neglected in (3.9), imply a deviation from pure thermal emission. If we consider the full consequences of energy conservation, now the distribution function for the emission of photons is not the standard Boltzmann distribution (2.4), but the distribution (see [17] - correcting the result in [14][15]-)

$$
<n(E)>=\frac{1}{\exp (2 \operatorname{Im} S)-1} \text {. }
$$

For our effective metric this can be written as

$$
<n(E)>=\frac{1}{\exp \left(4 \pi \int_{0}^{E} \frac{d E^{\prime}}{g\left(R_{+} ; M-E^{\prime}\right)}\right)-1}
$$

On the one hand, let us assume that a black hole has a mass $M$ and the effective metric does not posses a critical mass. Then, energy conservation would impose the range of energies for the emitted particles to be $0<E \leq M$.

On the other hand, let us assume that a black hole has a mass $M$ and the effective metric has a critical mass $M_{c r}<M$. If the energy of the emitted particle $E$ reached the value $E=M-M_{c r}$ then the black hole mass would be reduced to its critical mass and $g\left(R_{+} ; M-E\right)=g\left(R_{+} ; M_{c r}\right)=f^{\prime}\left(R_{+} ; M_{c r}\right)=0$. However, by definition of critical mass, $g\left(R_{+} ; M-E<M_{c r}\right)=f^{\prime}\left(R_{+} ; M-E<M_{c r}\right)$ is not defined. In this way, the distribution function in case a critical mass exists would be limited to a range of energies $0<E \leq M-M_{c r}$. Note that this already imposes energy conservation by forbidding the emitted quantum to carry more energy than the black hole mass.

In both cases, we see that the energy conservation approach tell us that a thermal spectrum, which would contain a tail of arbitrarily high energies, can not provide us with the correct spectrum.

Now, we would like to use this distribution in order to write the luminosity when energy conservation is taken into account. However, this requires the use of a grey-body factor that should take energy conservation into consideration. The correct grey-body factor can be found using (2.6) and takes the form (see [18])

$$
\gamma_{E C} \simeq 4 E^{2} R_{+}^{2}(M-E)
$$

where, as previously, $R_{+}(M-E)$ is $R_{+}$for a mass at infinity $M-E$.

If we now define

$$
\tilde{M}_{c r}=\left\{\begin{array}{lll}
0 & \text { if } \nexists M_{c r} \\
M_{c r} & \text { if } & \exists M_{c r}
\end{array},\right.
$$


we can use (3.10) and the greybody factor $\gamma_{E C}$ in order to write the luminosity when energy conservation is satisfied as

$$
\begin{array}{r}
L_{E C} \simeq \frac{1}{2 \pi} \int_{0}^{M-\tilde{M}_{c r}}<n(E)>\gamma_{E C} E d E \\
=\frac{1}{2 \pi} \int_{0}^{M-\tilde{M}_{c r}} \frac{4 E^{3} R_{+}^{2}(M-E)}{\exp \left(4 \pi \int_{0}^{E} \frac{d E^{\prime}}{g\left(R_{+} ; M-E^{\prime}\right)}\right)-1} d E
\end{array}
$$

\subsection{Preventing Black hole evaporation And explosions}

In subsection 2.2 we saw that the thermal approach implies that if close to zero mass

$$
\left.f^{\prime}\right\rfloor_{R_{+}} \simeq a M^{\alpha} \quad, \quad R_{+} \simeq b M^{\beta}
$$

where $2 \beta+4 \alpha<1, \beta>0$ and $\alpha \geq-1$, then the $\mathrm{BH}$ reaches its total evaporation (what includes the case of a final explosion). However, under the perspective of the energy conservation approach, we can now state a proposition specifically written to contrast with proposition 1 as follows.

Proposition 3 Let us consider an effective metric whose outer horizon exists for all $M>0$ with a non-zero surface gravity. If the metric satisfies around its zero mass

$$
\left.f^{\prime}\right\rfloor_{R_{+}} \simeq a M^{\alpha} \text { and } R_{+} \simeq b M^{\beta}
$$

where $2 \beta+4 \alpha<1, \beta>0$ and $\alpha \geq-1$, then a black hole satisfying energy conservation and modelled with this metric will not reach the total evaporation.

In order to show the proposition, it suffices to note that, when energy conservation is taken into account, one gets a luminosity (from (3.13))

$$
L_{E C} \simeq c M^{3+\alpha+2 \beta}
$$

where $c$ is a constant (see appendix C). The solution of

$$
\frac{d M}{d u}=-L_{E C}
$$

for the luminosity (3.15) with $^{5} \beta>0$ and $-1 \leq \alpha<1 / 4$ provides us with a mass evolution than only reaches a zero mass and, thus, absolute zero for $u \rightarrow \infty$. Therefore, when energy conservation is considered, the total evaporation is not reached.

Note, as a corollary, that a BH explosion will not happen. In particular, even considering the Schwarzschild solution $\left(\alpha_{S c h .}=-1 ; \beta_{S c h} .=1\right)$ one does not obtain an explosion when energy conservation is taken into account, but a final endless evaporation (see also [19]).

\footnotetext{
${ }^{5}$ What includes the case that reached the total evaporation in the thermal approach as a subcase.
} 


\subsection{Preventing Violations of the 3Rd LaW}

In subsection 2.3 we saw that the thermal approach implies a violation of the third law of black hole thermodynamics if

$$
\left.f^{\prime}\right\rfloor_{R_{+}} \simeq a\left(M-M_{c r}\right)^{\alpha}
$$

and $0 \leq \alpha<1 / 4$. However, if this model is considered under the perspective of the energy conservation approach we have the following

Proposition 4 Let us consider an effective metric possessing an outer horizon whose surface gravity is zero for a mass $M=M_{c r}>0$ while $R_{+}\left(M \geq M_{c r}\right)>0$. If the metric satisfies around the critical mass

$$
\left.f^{\prime}\right\rfloor_{R_{+}} \simeq a\left(M-M_{c r}\right)^{\alpha},
$$

where $0 \leq \alpha<1 / 4$, then the temperature of an evaporating black hole satisfying energy conservation and modelled with this metric never reaches absolute zero. In other words, the black hole does not violate the 3rd. law of black hole thermodynamics during its evaporation.

In order to show this, it suffices to note that, when energy conservation is taken into account, one gets a luminosity (from (3.13))

$$
L_{E C} \simeq c\left(M-M_{c r}\right)^{3+\alpha}
$$

where the constant $c$ takes the value (see appendix B)

$$
c=\frac{a R_{+}^{2}\left(M_{c r}\right)}{2 \pi^{2}}\left(\psi\left(\frac{4}{1-\alpha}\right)-3 \psi\left(\frac{3}{1-\alpha}\right)+3 \psi\left(\frac{2}{1-\alpha}\right)-\psi\left(\frac{1}{1-\alpha}\right)\right)
$$

and $\psi(z)$ is the digamma function. The solution of

$$
\frac{d M}{d u}=-L_{E C}
$$

for the luminosity (3.18) with $0 \leq \alpha<1 / 4$ provides now a mass evolution that only reaches the critical mass and, thus, absolute zero temperature for $u \rightarrow \infty$.

\section{Conclusions}

In this paper we have compared black hole evaporation from a strict thermal approach with the evolution obtained when energy conservation is satisfied. The explicit calculations have been carried out assuming the existence of general effective spherically symmetric spacetimes 
describing a quantum vacuum invariant under radial boosts (such as the ones found in, for example, [4][5][6][7][8][9]). Specifically, we have seen that one can construct models in the thermal approach that allow black hole explosions (propos. 1 with $-1 \leq \alpha<0$ ) or violations of the third law of black hole thermodynamics (propos.2). This contrasts with the models obtained when energy conservation is imposed since in this case the models, instead of exploding, asymptotically reach zero mass (propos.3) and instead of violating the 3rd law (in case a critical mass exists), form a remnant whose temperature only asymptotically approaches absolute zero (propos.4).

Let us comment that, in order to compute the luminosity, we have introduced a greybody factor since it is well-known that an important part of the total emitted radiation (up to 90\%) is later backscattered (see, for instance, [11]). However, this factor does not play an important role in preventing $\mathrm{BH}$ explosions or 3rd law violations. To see this, note that a calculation of the luminosity if the backscattered radiation were neglected $\left(\gamma_{E C} \sim 1\right)$ would have provide us with $L_{E C} \simeq \bar{c} M^{1+\alpha}(-1 \leq \alpha<1 / 4)$ replacing (3.15) and with $L_{E C} \simeq \bar{c}\left(M-M_{c r}\right)^{1+\alpha}(0 \leq \alpha<1 / 4)$ replacing (3.18), what again would have lead us to no black hole explosions in the first case ${ }^{6}$ and to no violations of the $3 \mathrm{rd}$ law in the second case.

In view of previously found links [20][21] between the fulfilment of the 3rd law under external influences and the fulfilment of some energy conditions, we would like to emphasize that our results on the fulfillment of the third law in $\mathrm{BH}$ evaporation do not require the imposition of any energy condition. This is absolutely appropriate since one expects the energy conditions to be violated in the evaporation process.

It has to be remarked that the calculations in this paper cannot be rigorous in the absence of a full Quantum Gravity Theory in which they could be strictly computed. Nevertheless, we have argued that energy conservation might be a sufficient condition for avoiding black hole explosions and violations of the third law. The physical mechanism behind this would be that, contrarily to the assumption in the thermal approach, energy conservation implies that a black hole cannot emit particles of arbitrary high energy. In this way, while the thermal approach is an excellent approximation for macroscopic black holes in which the back-reaction can be neglected it fails when applied in the last stages of black hole evaporation. On the other hand, the picture offered by the energy conservation approach suggests that in the last stages of the evaporation only long wavelength particles could tunnel out the horizon, what eventually would prevent black hole explosions and violations of the third law. Finally, it is interesting to note that, consequently, the energy conservation approach would also drastically limit the amount of information that can be carried away from the black hole in the last stages of its evaporation [18].

\footnotetext{
${ }^{6}$ Even if the possibility of a non-explosive total evaporation would exist for $-1 \leq \alpha<0$.
} 


\section{A. Some efFeCtive Metrics With A CRITICAL MASS}

Let us now describe, among the many examples that can be found in the literature, a couple of effective metrics possessing a critical mass:

- The renormalization group improved Schwarzschild solution. It was found by Bonanno and Reuter [5] and can be described by the metric (2.1) with

$$
f(R ; M)=1-\frac{2 G(R ; M) M}{R},
$$

where

$$
G(R ; M)=\frac{G_{0} R^{3}}{R^{3}+\tilde{\omega} G_{0}\left(R+\gamma G_{0} M\right)},
$$

$G_{0}$ is Newton's universal gravitational constant, $M$ is the mass measured by an observer at infinity and $\tilde{\omega}$ and $\gamma$ are constants coming from the non-perturbative renormalization group theory and from an appropriate cutoff identification, respectively.

As usual, the horizons in this solution can be found by solving $f(R ; M)=0$. The number of positive real solutions to this equation correspond to the positive real solutions of a cubic equation and depends on the sign of its discriminant or, equivalently, on whether the mass is bigger, equal or smaller than a critical value $M_{c r}$. In general, this critical value takes the form

$$
M_{c r}=a(\gamma) \sqrt{\frac{\tilde{\omega}}{G_{0}}}=a(\gamma) \sqrt{\tilde{\omega}} m_{p} \sim \sqrt{\tilde{\omega}} m_{p}
$$

where $m_{p}$ is Planck's mass.

If $M>M_{c r}$ then $f(R ; M)=0$ has two positive real solutions $\left\{R_{-}, R_{+}\right\}$satisfying $R_{-}<R_{+}$and $\kappa_{R_{+}}>0$. If $M=M_{c r}$ then there is only one positive real solution to the cubic equation $R_{+}$satisfying $\kappa_{R_{+}}=0$. If $M<M_{c r}$ the equation has not positive real solutions (thus one cannot define a surface gravity). In this way, by definition, the critical value $M_{c r}$ is a critical mass.

- Noncommutative black hole. The solution that we want to introduce can be found in [8][9] (Myung et al.) and is described by the metric (2.1) with

$$
f(R ; M)=1-\frac{4 M}{R \sqrt{\pi}} \gamma\left(\frac{3}{2}, \frac{R^{2}}{4 \theta}\right),
$$

where $M$ is the mass of the black hole, $\theta$ is a constant coming from the noncommutation of the geometry and the lower incomplete gamma function is defined by

$$
\gamma\left(\frac{3}{2}, \frac{R^{2}}{4 \theta}\right) \equiv \int_{0}^{\frac{R^{2}}{4 \theta}} r^{\frac{1}{2}} e^{-r} d r .
$$


As in the previous case, the number of solutions of $f(R ; M)=0$ depend on the value of the mass. If $M>M_{c r}=1.9 \sqrt{\theta}$ then two distinct horizons appear $\left\{R_{-}, R_{+}\right\}$satisfying $R_{-}<R_{+}$and $\kappa_{R_{+}}>0$. If $M=M_{c r}$ then there is only one positive real solution to the cubic equation $R_{+}$satisfying $\kappa_{R_{+}}=0$. If $M<M_{c r}$ the equation has not positive real solutions. In this way, $M_{c r}$ is a critical mass.

\section{B. Calculation of $L_{E C}$ and the third law}

We have to compute, for $M \sim M_{c r}$, the result of the integral

$$
L_{E C} \simeq \frac{1}{2 \pi} \int_{0}^{M-M_{c r}} \frac{4 E^{3} R_{+}^{2}(M-E)}{\exp \left(4 \pi \int_{0}^{E} \frac{d E^{\prime}}{g\left(R_{+} ; M-E^{\prime}\right)}\right)-1} d E
$$

with

$$
g\left(R_{+} ; M-E^{\prime}\right)=f^{\prime}\left(R_{+} ; M-E^{\prime}\right) \simeq a\left(M-E^{\prime}-M_{c r}\right)^{\alpha}
$$

and $0 \leq \alpha<1 / 4$. A change of variables $\left\{\mu=M-M_{c r}, x=E / \mu\right\}$ and the calculation of the corresponding integral allows us to write the argument in the exponential approximately as

$$
\delta \equiv \frac{4 \pi\left[1-(1-x)^{1-\alpha}\right]}{a(1-\alpha)} \mu^{1-\alpha} .
$$

Since $\delta$ is a small quantity for $\mu \sim 0$ one has $\exp (\delta)-1=\sum_{i \geq 1} \delta^{i} /(i !) \simeq \delta$. So that the luminosity near de critical mass $(\mu \sim 0)$ can be directly computed as

$$
L_{E C} \simeq \frac{a(1-\alpha) R_{+}^{2}\left(M_{c r}\right) I}{2 \pi^{2}} \mu^{3+\alpha}
$$

where

$$
I=\int_{0}^{1} \frac{x^{3} d x}{1-(1-x)^{1-\alpha}}=\frac{\psi\left(\frac{4}{1-\alpha}\right)-3 \psi\left(\frac{3}{1-\alpha}\right)+3 \psi\left(\frac{2}{1-\alpha}\right)-\psi\left(\frac{1}{1-\alpha}\right)}{1-\alpha} .
$$

Undoing the change of variables one directly obtains (3.18) with the sought constant $c$.

Let us remark that, while the calculation has been performed with the specific problematic range $0 \leq \alpha<1 / 4$ (treated in proposition 4 ) in mind, energy conservation prevents violations of the third law for $\alpha \geq 0$ (although the proof has to be modified for $\alpha \geq 1$ ). 


\section{Calculation of $L_{E C}$ and BH explosions}

We have to compute, for $M \sim 0$, the integral

$$
L_{E C} \simeq \frac{1}{2 \pi} \int_{0}^{M} \frac{4 E^{3} R_{+}^{2}(M-E)}{\exp \left(4 \pi \int_{0}^{E} \frac{d E^{\prime}}{g\left(R_{+} ; M-E^{\prime}\right)}\right)-1} d E
$$

with

$$
g\left(R_{+} ; M-E^{\prime}\right)=f^{\prime}\left(R_{+} ; M-E^{\prime}\right) \simeq a\left(M-E^{\prime}\right)^{\alpha},
$$

$R_{+}(M-E) \simeq b(M-E)^{\beta}, \beta>0$ and $-1 \leq \alpha<1 / 4$. Defining $x \equiv E / M$ we can proceed exactly as in appendix B obtaining the sought result

$$
L_{E C} \simeq \frac{a b^{2}(1-\alpha) I}{2 \pi^{2}} M^{3+\alpha+2 \beta}
$$

where

$$
I=\int_{0}^{1} \frac{x^{3}(1-x)^{2 \beta} d x}{1-(1-x)^{1-\alpha}} .
$$

Note that, while the calculation has been performed with the specific problematic range $-1 \leq \alpha<1 / 4$ (treated in proposition 3) in mind, energy conservation prevents the total evaporation for $\alpha \geq-1$ (although the proof has to be modified for $\alpha \geq 1$ ).

\section{REFERENCES}

[1] Hawking S W 1975 Commun. Math. Phys. 43199

[2] Parikh M K and Wilczek F 2000 Phys. Rev. Lett. 855042 (arXiv:hep-th/9907001v3)

[3] Grøn Ø 1986 Am. J. Phys. 5446

[4] Emparan R, Horowitz G T and Myers R C 2000 Phys. Rev. Lett. 85499

[5] Bonanno A and Reuter M 2000 Phys. Rev. D 62043008

[6] Dymnikova I G 1992 Gen. Rel Grav. 24235

[7] Dymnikova I G 2000 Phys. Lett. B 47233

[8] Nicolini P 2009 Int. J. Phys. A 241229

[9] Myung Y S, Kim Y-W and Park Y-J 2007 J. High Energ. Phys. 02012 
[10] Hawking S W and Ellis G F R 1973 The large scale structure of space-time. Cambridge: Cambridge University Press

[11] Fabbri A and Navarro-Salas J 2005 Modeling Black Hole Evaporation London: Imperial College Press

[12] Harmark T, Natario J and Schiappa R 2010 Adv. Theor. Math. Phys. 14727 (arXiv:hepth/0708.0017)

[13] Painlevé P 1921 C. R. Acad. Sci. (Paris) 173677

[14] Kraus P and Wilczeck F 1995 Nucl. Phys. B433 403 (arXiv:hep-th/9408003)

[15] Kraus P and Wilczeck F 1995 Nucl. Phys. B437 231 (arXiv:hep-th/9411219)

[16] Israel W and Yun Z 2010 Phys. Rev. D 82124036

[17] Keski-Vakkuri E and Kraus P 1997 Nucl. Phys. B491 249 (arXiv:hep-th/9610045)

[18] Torres R, Fayos F and Lorente-Espín O 2013 Phys. Lett. B 720198

[19] Torres R, Fayos F and Lorente-Espín O 2013 Int. J. Mod. Phys. D 221350037

[20] Israel W 1986 Phys. Rev. Lett. 57397

[21] Jacobson T and Sotiriou T P 2009 Phys. Rev. Lett. 103141101 